\title{
Local Modulation of Basal Forebrain: Effects on Working and Reference Memory
}

\author{
Bennet Givens and David S. Olton \\ Department of Psychology, The Johns Hopkins University, Baltimore, Maryland 21218
}

The functional roles of the medial septal area (MSA) and nucleus basalis magnocellularis (NBM) in memory were investigated to determine (1) their relative contribution to working and reference memory, (2) their operation in spatial and nonspatial memory, (3) the temporal dynamics of the neural activity within these nuclei as they relate to mnemonic processes, (4) the neurochemical regulation of their activity, and (5) the importance of $\mathrm{ACh}$ for their function. Working memory was tested in a continuous conditional discrimination (CCD), and reference memory was tested in the CCD and a sensory discrimination (SD). Bipolar recording electrodes in the dentate hilus monitored hippocampal EEG (theta rhythm). Immediately prior to behavioral testing, trained rats were infused with tetracaine, scopolamine, or muscimol into the MSA or NBM, and the subsequent behavioral and physiological changes were measured and correlated. MSA infusions of all three drugs reduced the power of hippocampal theta and impaired choice accuracy in the CCD; the magnitude of both effects was greater for larger doses and steadily decreased over time after the infusion, producing a strong positive correlation between the power of theta and choice accuracy in the CCD. These infusions had no effect on measures of reference memory in the CCD or in the SD. The results demonstrate that rhythmic activity along the septohippocampal pathway reflects processing of nonspatial working, but not reference memory. NBM infusions did not affect hippocampal theta but did reduce choice accuracy in the SD and completely disrupted performance in the CCD. The NBM appears to have a critical role in both working and reference memory.

[Key words: hippocampus, scopolamine, GABA, ACh, theta rhythm, nucleus basalis, basal forebrain, memory]

The two principal components of the basal forebrain, the medial scptal arca (MSA) and nuclcus basalis magnocellularis (NBM), provide the major cholinergic innervation to the hippocampus and neocortex, respectively. The MSA and its cholinergic projections to the hippocampus and related temporal lobe structures are critical for trial-dependent working memory (Olton et

\footnotetext{
Received May 10, 1993; revised Oct. 4, 1993; accepted Nov. 17, 1993.

We thank G. Wenk and I. Malak for assistance with ${ }^{3} \mathrm{H}$-hemicholinium-3 binding assays, M. Wagster for assistance with ${ }^{3} \mathrm{H}$-muscimol autoradiography, K. Pang, A. Markowska, and M. Pontecorvo for discussions of experimental design and analysis, and $M$. Barra and $M$. Conroy for assistance with the bchavioral cxperiments. We also acknowledge $A G-10015$ to D.S.O. from the National Institute of Aging and NS-08616 to B.G. for support of the research.

Correspondence should be addressed to Bennet Givens, Department of Psychology, The Ohio State University, Columbus, OH 43210.

Copyright (C) 1994 Society for Neuroscience $0270-6474 / 94 / 143578-10 \$ 05.00 / 0$
}

al., 1992). The hypothesis that the septohippocampal system is required for working memory, but not for trial-independent reference memory, was tested in the present experiment using two discriminations that were identical in all respects (stimuli, responses, reinforcements) except that one task required working memory and the other did not.

Basal forebrain neural activity is modulated by afferent input. Two important regulators of basal forebrain activity are GABA and ACh. Muscimol, a $\mathrm{GABA}_{\mathrm{A}}$ receptor agonist, and scopolamine, a muscarinic antagonist, inhibit MSA neural activity. Intraseptal infusions of muscimol suppressed hippocampal theta rhythm and impaired performance on a variety of spatial working memory tasks (Chrobak et al., 1989; Brioni et al., 1990; Givens and Olton, 1990; Chrobak and Napier, 1992; Nagahara and McGaugh, 1992). Intraseptal infusion of scopolamine (15 $\mu \mathrm{g}$ ) disrupts the rhythmic output from MSA neurons and impairs working memory (Givens and Olton, 1990). The behavioral and electrophysiological consequences of transmitter-specific regulation of basal forebrain were further investigated in the present experiments by using site-directed microinfusions of GABAergic and cholinergic compounds to determine the effect on neural, temporal, and mnemonic parameters.

Whereas spatial functions of the hippocampus in the rat have been well established, nonspatial functions are still being debated (see Barnes, 1988; see also Hippocampus, Vol 1, No 3, 1991). In the present experiment, an operant procedure that assessed working memory was designed so that the relevant dimensions of the stimuli were nonspatial. If the MSA is required for nonspatial working memory as well as spatial working memory, then infusions of scopolamine and muscimol into the MSA should impair choice accuracy in this nonspatial working memory task.

Hippocampal theta rhythm may have an important role in mnemonic processes. The theta rhythm is dependent on the septohippocampal pathway and has been linked to learning and memory (Landfield et al., 1971; Winson, 1978; Rawlins, 1985). Theta rhythm and working memory are correlated such that decreases in theta power are associated with decreases in choice accuracy (Givens and Olton, 1990). Although these studies have established a global correlation between theta and memory, the precise moment-to-moment association between the two measures has not been investigated. The present experiment recorded hippocampal theta during the entire test session so that theta could be directly correlated with choice accuracy. If hippocampal theta itself or neural mechanisms associated with theta are involved in working memory, then choice accuracy in working memory tasks should be correlated with theta, and the reduction in choice accuracy produced by MSA infusions of 
muscimol and scopolamine should be correlated with the magnitude of reduction in the power of theta.

The cholinergic nature of basal forebrain projections may be critical for their function. In large doses (100-300 ng) that also reduce hippocampal high-affinity choline uptake, intraseptal muscimol impaired spatial learning and increased extinction responding (Blaker et al., 1984; Brioni et al., 1990). However, lower doses can reduce theta and impair spatial working memory, but no information is available to determine if they also decrease cholinergic indices in the hippocampus. The present experiment tested the hypothesis that the decrease in choice accuracy in working memory tasks produced by intraseptal muscimol is due to inhibition of cholinergic activity in the hippocampus, as measured by ${ }^{3} \mathrm{H}$-hemicholinium- 3 binding. If the cholinergic system is involved in the working memory impairments following these MSA infusions, then ${ }^{3} \mathrm{H}$-hemicholinium-3 binding in the hippocampus should be reduced by the same doses that impair working memory.

The other principal component of the basal forebrain, the nucleus basalis magnocellularis (NBM), has been associated with arousal, motivation, attention, and memory (Olton and Wenk, 1987; Buzsaki et al., 1988; Richardson and DeLong, 1990). The NBM has widespread projections throughout the cerebral cortex (Mesulum et al., 1983) and is regulated by a number of afferent inputs, including GABAergic afferents from nucleus accumbens and cholinergic afferents from the brainstem (7áborszky et al., 1991). Studies that have used neurotoxic lesions suggest that the NBM may be critically involved in reference memory (for review, see Olton and Wenk, 1987), although these deficits may involve other brain structures (Dunnett et al., 1991), and other cognitive processes such as attention (Robbins et al., 1989). Very low doses of muscimol infused into the NBM can impair conditional visual discrimination (Dudchenko and Sarter, 1991; Muir et al., 1992). If the NBM is important for trial-independent reference memory, and if GABAergic and cholinergic afferents are important regulators of NBM neural activity, then muscimol or scopolamine infused into the NBM will impair performance on both the working and reference memory tasks.

The present study investigates the time dependence of GABAergic and cholinergic regulation of basal forebrain as it relates to nonspatial working and reference memory. The possibility of a functional dissociation between MSA and NBM was tested by infusing muscimol and scopolamine in the NBM and comparing the behavioral effects with those following infusions into the MSA. If activity in the septohippocampal pathway is critical for the timing of working memory processes, then the electrophysiological and behavioral consequences of intraseptal microinfusions should be highly correlated. The results indicate that both muscimol and scopolamine equally disrupt septohippocampal neural activity, and impair mnemonic performance in a working but not in a reference memory task, and that the electrophysiological changes correlate with errors in working memory. Results from experiments using NBM microinfusions indicate that the NBM may contribute to both working and reference memory.

\section{Materials and Methods}

\section{Subjects}

Male Long-Evans hooded rats (Charles River) were virus antibody free and weighed $300-400 \mathrm{gm}$. The colony room had a 12:12 hr light : dark cycle (lights on at 7:00 A.M.) and was maintained at $25^{\circ} \mathrm{C}$. During the first week, food and water were available ad libitum, after which only food was available ad libitum in the home cage. Each rat was handled for $3 \mathrm{~d}$ before behavioral testing began.

The 42 rats were divided into three groups. Two groups received behavioral testing (18 in the CCD, 14 in the SD) followed by histology ( 29 with dye infusions, 3 with ${ }^{3} \mathrm{H}$-muscimol infusions). The third group $(N=10)$ was used for ${ }^{3} \mathrm{H}$-hemicholinium-3 autoradiography.

\section{Apparatus}

Behavior. The operant chambers used for training were each $26 \times 26$ $\times 30 \mathrm{~cm}$ and had four walls and a hinged ceiling made of $0.5-\mathrm{cm}$-thick Plexiglas. The floor consisted of parallel stainless steel rods $(0.5 \mathrm{~cm}$ diameter), $2 \mathrm{~cm}$ apart. A cup for delivery of water was located in the center of the front panel, $5 \mathrm{~cm}$ above the floor. Two response levers, one on each side of the front panel and $5 \mathrm{~cm}$ from the cup, were positioned $4 \mathrm{~cm}$ above the floor. A tone generator and a light were located $14 \mathrm{~cm}$ and $17 \mathrm{~cm}$ above the water reward cup, respectively. The operant chamber was placed in a box $(40 \times 40 \times 50)$. All stimuli were controlled and responses recorded with an IBM personal computer using software developed by Med Associates (East Fairfield, VT).

The operant chamber used for electrophysiological testing was identical to the training chambers except for a commutator attached to the ceiling, electrical shielding, and acrylic rods for the floor.

Surgery. A Knopf (Tejunga, CA) stereotaxic apparatus was used for surgery. Each cannula was 26 gauge stainless steel hypodermic tubing, $10 \mathrm{~mm}$ long. Each stylet used to occlude the cannula was 32 gauge wire, $11 \mathrm{~mm}$ long, with the last $1 \mathrm{~mm}$ bent at a $110^{\circ}$ angle. The electrodes for recording hippocampal theta were parallel stainless steel wires, 76.2 $\mu \mathrm{m}$, coated with Teflon (A-M Systems, Everett, WA). The reference electrode was a single $254 \mu \mathrm{m}$ stainless steel wire insulated to the tip with Teflon.

Intracranial infusions. Intracranial drug infusions used a Sage microsyringe pump (Orion Res. Inc., Boston, MA). An injector made of 32 gauge stainless steel tubing, approximately $30 \mathrm{~mm}$ long, bent $12 \mathrm{~mm}$ from the end, was glued to polyethylene tubing (PE-10, Intramedic) that was connected to a $10 \mu \mathrm{l}$ Hamilton syringe mounted on the infusion pump. Tetracaine hydrochloride $(20 \mu \mathrm{g} / \mu \mathrm{l})$, muscimol $(10,30$, and 60 $\mathrm{ng} / \mu \mathrm{l})$, and scopolamine hydrochloride $(10,30$, and $60 \mu \mathrm{g} / \mu \mathrm{l})$ were prepared in saline and stored frozen $\left(-40^{\circ} \mathrm{C}\right)$ at their final concentrations in $100 \mu$ aliquots. Doses were based on the salt of the drug.

Electrophysiological recording. An operational amplifier sent unity gain signals through a slip ring commutator (Biela, Anaheim, CA) to amplifiers, filters, and an analog-to-digital acquisition board. The digitized signals were stored and analyzed by software developed by Brainwave, Inc. (Thorton, CO).

\section{Procedure}

Shaping. After $1 \mathrm{~d}$ of water restriction, each rat was shaped to press each lever for a water reward $(\approx 0.1 \mathrm{ml})$. Each session was 90 min long. One session was given each day until 100 presses were made in a session.

Continuous conditional discrimination ( $C C D$ ). The $C C D$ is a modified version of the continuous nonmatch-to-sample task described previously by Pontecorvo (1983), and characterized as hippocampally dependent by Wan et al. (1990). For each trial, either a light or a tone was presented. If the stimulus on the current trial was the same as the stimulus on the previous trial (match trial), responses on one lever was reinforced. If the stimulus on the current trial was different from that presented on the previous trial (a nonmatch trial), responses on the other lever were reinforced. A correct response was followed by delivery of water $(0.1 \mathrm{ml})$. The stimulus was turned off following a lever press, or after $5 \mathrm{sec}$ if no lever press occurred. The interstimulus interval (ISI) was $2.5,10$, or $20 \mathrm{sec}$; the three ISIs were presented randomly with the constraint that within each block of 15 trials, each ISI occurred five times. One session, $90 \mathrm{~min}$ long, was given each day, $7 \mathrm{~d}$ a week.

$\Lambda$ sequence of match trials was alternated with a sequence of nonmatch trials. The number of trials in each sequence was $41,21,11,5$, and 3 . Training in each sequence continued until a criterion of a mean choice accuracy of $80 \%$ on both match and nonmatch trials was obtained for 2 consecutive days, and then the next shorter sequence was begun. After criterion performance on sequences of three, the presentation of match and nonmatch trials was randomized, and training continued to the same criterion of performance.

Sensory discrimination ( $S D$ ). Each trial began with the presentation of one stimulus, either a light or a tone. For a light stimulus, pressing the right lever was the correct response. For a tone stimulus, pressing 
A.

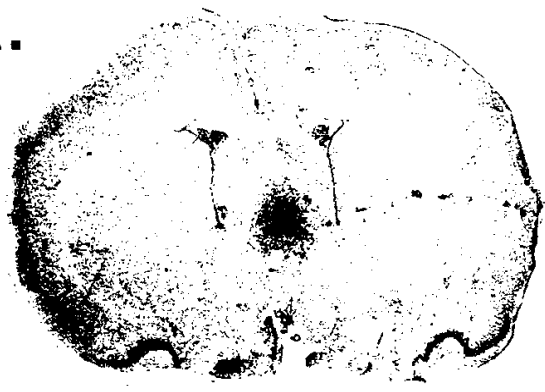

B.
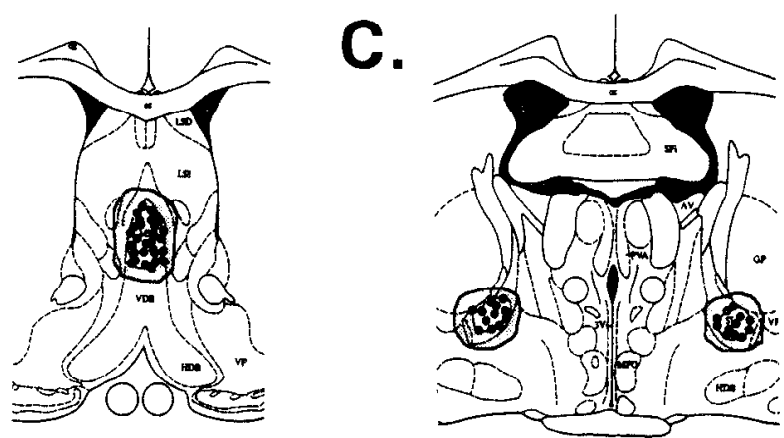

Figure 1. Location of intracranial sites of infusion. $A$, Distribution of tritiated muscimol $10 \mathrm{~min}$ after infusion into the MSA. A coronal section at the level of the MSA was stained with Nissl for cell bodies and autoradiography of ${ }^{3} \mathrm{H}$-muscimol. Note that the tritiated muscimol is concentrated in the MSA and that the cannula tract is visible in the overlying cortex. $B$, Location of cannula tips $(\bullet)$ in the MSA collapsed $\pm 600 \mu \mathrm{m}$ onto a representative coronal section, $700 \mu \mathrm{m}$ anterior to bregma. $C$. Location of bilateral cannula tips ( $)$ in the NBM collapsed $\pm 600 \mu \mathrm{m}$ onto a single coronal section, $400 \mu \mathrm{m}$ posterior to bregma. In both $B$ and $C$ the thick outer line represents the maximum extent of dye diffusion, and the thin inner line is the minimum overlap from all dye infusions. The region between the two lines is shaded. The coronal sections were adapted with permission from Paxinos and Watson (1986).

the left lever was the correct response. A response turned off the stimulus and began a variable ISI of $2.5,10$, or $20 \mathrm{sec}$. A correct response was followed by water $(0.1 \mathrm{ml})$. If after $5 \mathrm{sec}$ a lever was not pressed, the stimulus was turned off and the ISI for the next trial began. One session, $90 \mathrm{~min}$ long, was given each day, $7 \mathrm{~d}$ a week. Training continued until a mean of $80 \%$ correct responding was obtained for each stimulus for three consecutive sessions.

Three dependent variables were measured in both behavioral tasks. Choice accuracy was the percentage of total responses that were correct, response omissions were the percentage of trials in which no response was made, and ISI responses were the number of responses during the ISI.

Surgery. After anesthesia with pentobarbital sodium $(45 \mathrm{mg} / \mathrm{kg})$, each rat was placed in the stereotaxic apparatus. The scalp was retracted and holes were drilled through the skull to implant guide cannulas, electrodes, and jeweler's screws. For MSA infusions, the guide cannula was placed $0.6 \mathrm{~mm}$ anterior and $1.5 \mathrm{~mm}$ lateral to bregma at a $15^{\circ}$ angle toward the midline. The tip of the cannula was placed $5.7 \mathrm{~mm}$ below the dura. For NBM infusions, two guide cannulas were placed bilaterally, $1.5 \mathrm{~mm}$ posterior and $2.0 \mathrm{~mm}$ lateral to bregma. The end of each cannula was $9 \mathrm{~mm}$ below the dura. $\Lambda$ recording electrode was placed $3.5 \mathrm{~mm}$ posterior and $2.5 \mathrm{~mm}$ lateral to bregma, with the tip $3 \mathrm{~mm}$ below the dura. A reference electrode was placed in the contralateral parietal cortex. The electrodes were atlached to a connector that was mounted on the skull over the cerebellum. The cannula, electrodes, and connector were fixed into place with dental acrylic. The incision was infiltrated with topical lidocaine (5\%), and the rat was placed in a warm environment until all reflexes and motor abilities were normal.

Postoperative behavior. Four days after surgery, testing with the same procedure used at the end of the preoperative testing (randomized sequence of match and nonmatch trials) was begun in the recording cham- ber. Testing continucd for at lcast $4 \mathrm{~d}$ and until the preoperative criterion of performance was attained. Performance in the recording chamber was not significantly different from performance in the training chamber $(p>0.05)$. The mean $( \pm$ SEM) number of trials/session was $274( \pm 28)$ and $321( \pm 24)$ in the CCD and SD, respectively.

Microinfusion. Each rat was placed on a clean, padded platform. The stylet in the cannula was removed and immediately rinsed with $80 \%$ ethanol. A sterile injector was placed into the guide cannula, and the syringe pump was turned on to deliver fluid at $0.1 \mu \mathrm{l} / \mathrm{min}$. The rat was free to move about the platform while the movement of an air bubble, $0.01 \mu \mathrm{l}$ in the tubing between the drug and the deionized water, was monitored to assure proper drug delivery. After $5 \mathrm{~min}$, the syringe pump was turned off. After an additional $1 \mathrm{~min}$ to allow for diffusion of drug away from the injector tip, the injector was removed and the stylet reinserted into the guidc cannula. The bilatcral NBM infusions were made sequentially. Each rat was immediately put in the recording chamber for behavioral testing.

Microinfusion took place no more than twice a week with at least 2 d separating infusions. Control performance was determined by averaging data from sessions that occurred on the day $(24 \mathrm{hr})$ before drug testing. The order of drug, dose, and site of injection was completely randomized across the entire study. With two exceptions, each rat received each dose of each drug. The drugs and doses were saline $(0.5 \mu 1)$, tetracaine $(10 \mu \mathrm{g})$, muscimol $(5,10$, and $30 \mathrm{ng})$, and scopolamine $(5$, 15 , and $30 \mu \mathrm{g}$ ).

Hemicholinium binding. The effect of intraseptal muscimol on presynaptic uptake of choline, an index of cholinergic function, was assessed in 10 rats. Ten minutes after microinfusion of saline or muscimol (15 ng) into the MSA, each rat was decapitated. The brain was rapidly removed and dissected into frontal cortex, hippocampus, entorhinal cortex, and striatum. Each section was rapidly frozen on dry ice and stored at $-80^{\circ} \mathrm{C}$.

Tissue was homogenized in $0.32 \mathrm{M}$ sucrose and centrifuged $(1000 \times$ $g$ ) for $15 \mathrm{~min}$ at $4^{\circ} \mathrm{C}$. The supernatant was centrifuged $(36,000 \times g)$ for $20 \mathrm{~min}$ at $4^{\circ} \mathrm{C}$, and then the pellet was resuspended in $10 \mathrm{~mm} \mathrm{Na}-\mathrm{K}$ phosphate buffer, containing $150 \mathrm{~mm} \mathrm{NaCl}, \mathrm{pH} 7.4$. Binding was determined in triplicate with $2.3 \mathrm{nM}^{3} \mathrm{H}$-hemicholinium-3, and nonspecific binding in the presence of $1 \mu \mathrm{M}{ }^{3} \mathrm{H}$-hemicholinium-3. The reaction was incubated at $25^{\circ} \mathrm{C}$ for $25 \mathrm{~min}$. The membranes were collected on a cell harvester. Radioisotope emissions were counted on a scintillation counter, and a Lowery protein assay was performed on the supernatant.

\section{Data analysis}

The data were analyzed as a repeated measure over time. The five independent variables were task, drug, time during session, delay, and site of infusion. The time during session was nine blocks of $10 \mathrm{~min}$ each. The tasks were CCD or SD. Drugs were saline, tetracaine, muscimol (three doses), and scopolamine (three doses). Delays were 2.5, 10 , and $20 \mathrm{sec}$. Sites of infusion were MSA and NBM.

Behavior. Dependent variables were choice accuracy, response omissions, and ISI responses. For each behavioral variable, a MANOVA was calculated followed by univariate ANOVAs to determine the source of the main effects, and post hoc Newman-Keuls analysis when appropriate. For the CCD, the MANOVA was time $(9) \times$ drug $(9) \times$ delay (3). For the SD, the MANOVA was time (9) $\times$ drug $(3) \times$ site of infusion (2). An additional analysis of behavioral variables as a function of cannula tip location was performed by $t$ test.

Physiology. The dependent variable was mean spectral power in the theta frequency $(6-8 \mathrm{~Hz})$. The mean power of theta was determined for each block of $10 \mathrm{~min}$ during the $90 \mathrm{~min}$ session. The statistical analyses were the same as those used for the behavioral data. In addition, a regression analysis was calculated on the mean choice accuracy and mean theta power across time for each ISI.

Neurochemistry. Differences in hemicholinium binding in each of the four brain regions were evaluated by $t$ test for a comparison of the means for muscimol infusion and saline infusion.

\section{Histology}

Chicago sky blue dye $(0.5 \%$ in $0.5 \mu \mathrm{l}$ water) was infused into the MSA in 29 rats and in the NBM in 12 of these rats. After $10 \mathrm{~min}$, the rat was decapitated and the brain removed. After 1 week of fixation in formalin, and $24 \mathrm{hr}$ in sucrose, the brain was frozen and cut into coronal sections, $40 \mu \mathrm{m}$ thick, from the anterior MSA through the dorsal hippocampus. Each section was stained with Congo red. The location of the tip of 


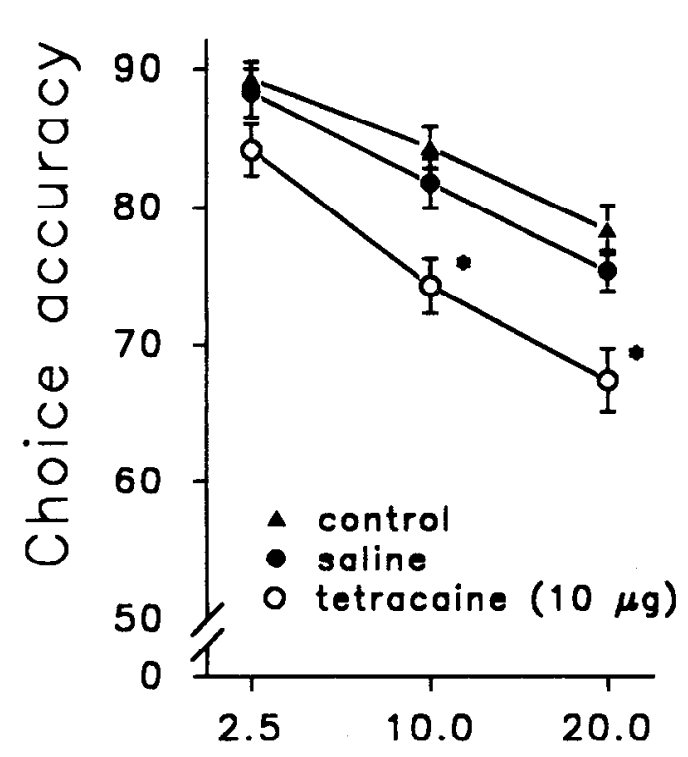

\section{Interstimulus interval (sec)}

Figure 2. Choice accuracy in the continuous conditional discrimination (CCD) as a function of delay interval following intraseptal infusions. Saline and low doses of muscimol and scopolamine had no effect on choice accuracy. Tetracaine and higher doses of muscimol and scopolamine produced a dose- and delay-dependent reduction in choice accuracy. ${ }^{*}, p<0.05 ; \dagger, p<0.01$; compared to saline.

each cannula and electrode, and the extent of dye diffusion were recorded. The MSA and NBM were divided into dorsoventral and mediolateral segments for analysis of behavioral effects as a function of infusion site.

${ }^{3} \mathrm{H}$-muscimol was infused into the MSA in order to determine the extent of diffusion of the drug from the injection site in three rats. Muscimol (30 ng in $0.5 \mu \mathrm{l} ; 10 \mathrm{nCi}$ of ${ }^{3} \mathrm{H}$-labeled muscimol) was infused as described above. Ten minutes after infusion, the rat was decapitated and the brain was rapidly removed and frozen in isopentane. The brain was cut into $20 \mu \mathrm{m}$ thin sections. Each section was thaw-mounted onto gelatin-coated slides, desiccated for $24 \mathrm{hr}$ at $0-4^{\circ} \mathrm{C}$, and placed on tritium-sensitive film in an autoradiographic cassette for 2 months. The film was developed by standard photographic techniques.

\section{Results}

Histology

All 32 MSA infusions included the MSA; five also extended into the intermediate division of the lateral septum. The autoradiographic distribution of tritium in the MSA of one rat that received ${ }^{3} \mathrm{H}$-muscimol is presented in Figure $1 \mathrm{~A}$. The location of each cannula tip and the extent of dye diffusion are shown in Figure $1 B$.

All 12 NBM dye infusions included the NBM; three also extended into the globus pallidus, just dorsal to the NBM. The location of each cannula tip and extent of dye diffusion are shown in Figure $1 C$.

\section{Continuous conditional discrimination (CCD)}

The mean $( \pm$ SEM) number of sessions required to complete the five stages of training was $64( \pm 8)$. The most difficult transition was the last step to random presentation of match and nonmatch trials, which took $32( \pm 12)$ sessions.

Postoperative performance under control conditions was delay dependent; that is, the mean choice accuracy decreased as the interstimulus interval (ISI) increased $\left(F_{2,126}=4.53, p<0.05\right.$;
Fig. 2). The mean control level of choice accuracy was 89.3 $( \pm 1.2), 84.4( \pm 1.5)$, and $78.4( \pm 1.8)$, respectively, for 2.5, 10, and $20 \mathrm{sec}$ ISIs.

Measures of general task performance were highly consistent within subjects across daily sessions. Responding during the ISI was low, with a mean of $0.8( \pm 0.7)$ responses per trial during control sessions. Response omissions were infrequent, occurring on $8.0 \%( \pm 3.6 \%)$ of trials. The omissions usually occurred at the end of a session, and probably were due to satiation because rats received most of their daily water in the task.

\section{MSA infusions}

Behavior. A total of 104 infusions were given to 18 rats $(5.8 \pm$ 1.2 infusions/rat). Saline had no effect on choice accuracy, indicating that the infusion procedure itself did not alter behavior (Fig. 2).

Choice accuracy was significantly affected by drug infusions (main effect of drug, $F_{8,242}=5.97, p<0.01$; Fig. 2), and was dependent on the length of the delay interval, with longer ISIs resulting in lower choice accuracy (main effect of delay, $F_{2,242}=$ $3.26, p<0.05$ ). Moreover, the magnitude of the drug-induced impairment increased as the ISI increased (drug $\times$ delay interaction, $p<0.01$ ).

The time of testing after infusion also affected choice accuracy (main effect of timc, $F_{8.242}=2.86, p<0.05$ ). The drug-induced decreases in choice accuracy diminished as the time in the test session increased (drug $\times$ time interaction, $p<0.01$ ). This interaction was dependent on delay interval, with longer delays being more affected by drug and time (delay $\times$ drug $\times$ time interaction, $p<0.05$ ). The time-dependent effects of drug infusion are illustrated in Figure 3, in which the results over time from the largest dose of each drug at three different delays are shown. 


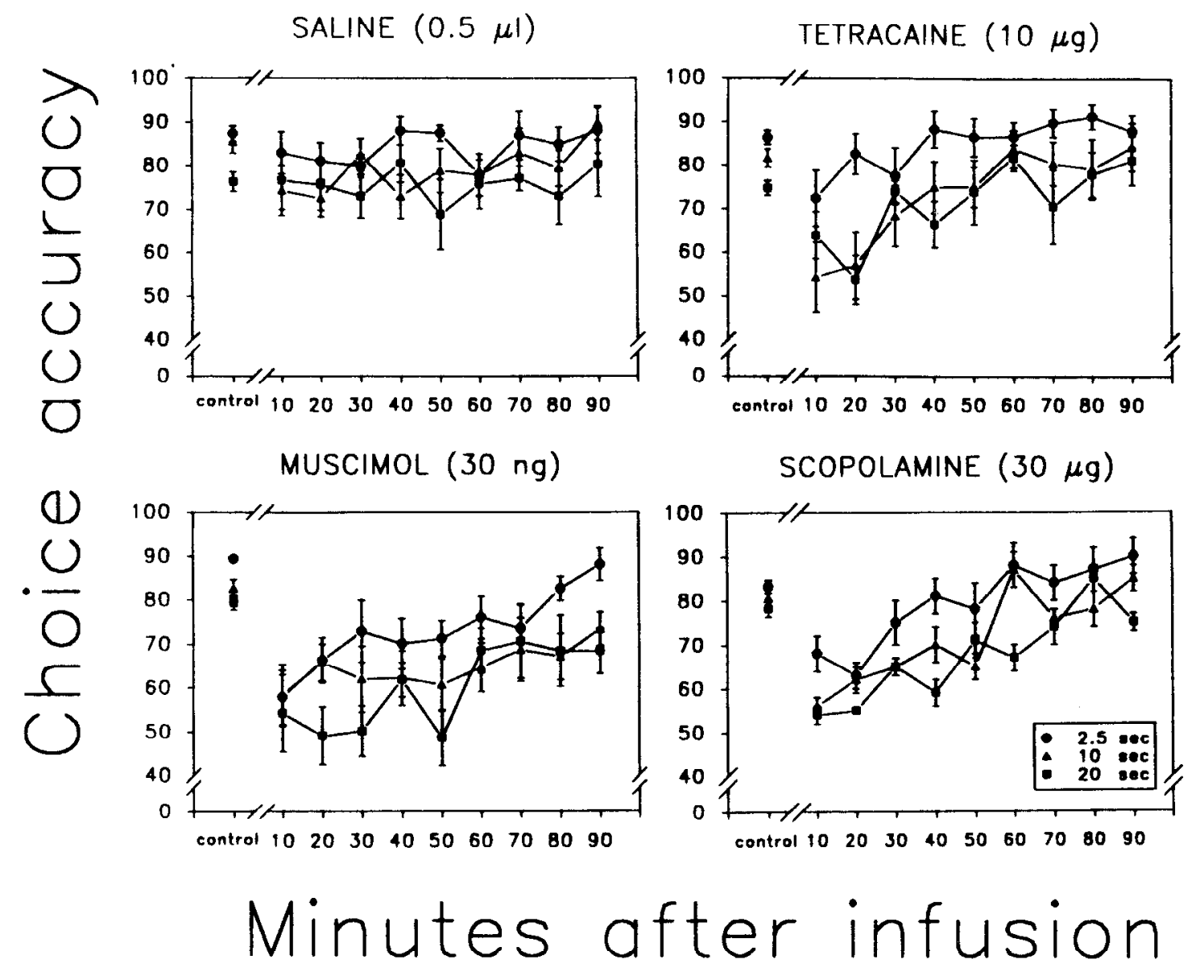

Figure 3. Intraseptal drug infusions decreased choice accuracy in the $\mathrm{CCD}$. The control choice accuracy (left side of each panel) was from sessions the day before infusion sessions. Drug, delay, and time after infusion had significant effects on choice accuracy. See text for an explanation of time course of drug effects.

Saline, when compared to control, produced no significant effect on choice accuracy ( $p>0.05$; Fig. 2$)$, which remained stable throughout each session (Fig. 3, top left).

Tetracaine $(10 \mu \mathrm{g})$ impaired choice accuracy at the 10 and 20 but not the $2.5 \mathrm{sec}$ ISI $(p<0.01$; Fig. 2). During the first 30 min, tetracaine impaired mean choice accuracy at all three ISIs (Fig. 3, top right), after which choice accuracy rapidly improved and remained within baseline levels for the remainder of the session.

Muscimol produced a dose- and delay-dependent impairment of choice accuracy. The $5 \mathrm{ng}$ dose had no effect at any time or delay $(p>0.05)$, the $10 \mathrm{ng}$ dose had a greatcr cffect on the longer delays ( $p<0.05$ at $2.5 \mathrm{sec}, p<0.01$ at 10 and $20 \mathrm{sec}$ ISIs), and the $30 \mathrm{ng}$ dose had an effect at all three ISIs $(p<$ 0.01; Fig. 2). Muscimol (30 ng), at the start of testing, reduced mean choice accuracy to chance levels $(p<0.01)$. Choice accuracy returned to baseline levels between 30 and 90 min postinfusion, with the shortest delay recovering first, followed by the middle delay. Choice accuracy at the longest delay was still below baseline $90 \mathrm{~min}$ after infusion (Fig. 3, bottom left).

Scopolamine produced a pattern of results similar to that for muscimol. The low dose $(5 \mu \mathrm{g})$ had no significant effect at any time or delay $(p>0.05)$. The intermediate dose $(15 \mu \mathrm{g})$ significantly decreased choice accuracy at the 10 and 20 but not the $2.5 \mathrm{sec}$ ISI $(p<0.01 ;$ Fig. 2$)$. The largest dose $(30 \mu \mathrm{g})$ reduced choice accuracy at all three ISIs $(p<0.01$ ), reducing accuracy to chance levels during the first $20 \mathrm{~min}$, followed by a gradual delay-dependent recovery. The recovery of performance after scopolamine was more rapid and complete when compared to muscimol (Fig. 3, bottom right).

Measures of general task performance were not significantly altered: response omissions $\left(F_{8,1 / 6}=0.83, p>0.05\right)$, response bias $\left(F_{8.176}=0.64, p>0.05\right)$, and ISI responses $\left(F_{8,176}=1.2, p\right.$ $>0.05$ ). Analysis of behavioral data as a function of location of infusion revealed no correlation between the site of infusion and performance on any dimension $(p>0.05)$.

Theta. Reliable hippocampal theta was obtained from 16 of the 18 rats that completed behavioral testing. Drug infusion significantly suppressed theta activity (main cffect of drug, $F_{8,147}$ $=8.7, p<0.01$ ). The magnitude of the suppression decreased as time in the test session increased (main effect of time after infusion, $F_{8.147}=4.3, p<0.01$ ). Saline had no effect on theta activity at any time after infusion. Figure 4 , taken from the same sessions as Figure 3, shows that tetracaine, muscimol, and scopolamine significantly suppressed theta activity (all $p<0.05$ ).

Tetracaine $(10 \mu \mathrm{g})$ abolished the hippocampal theta rhythm during the first $40 \mathrm{~min}(p<0.01)$, after which theta rapidly returned to baseline (Fig. 4). Muscimol produced a dose-dependent disruption of theta, and at $30 \mathrm{ng}$ significantly suppressed theta across the entire 90 min session ( $p<0.01$; Fig. 4). Likewise, scopolamine disrupted theta in a dose-dependent manner, with $30 \mu \mathrm{g}$ suppressing theta throughout the first $50 \mathrm{~min}(p<$ 0.01; Fig. 4).

Theta and choice accuracy. Choice accuracy and theta were 


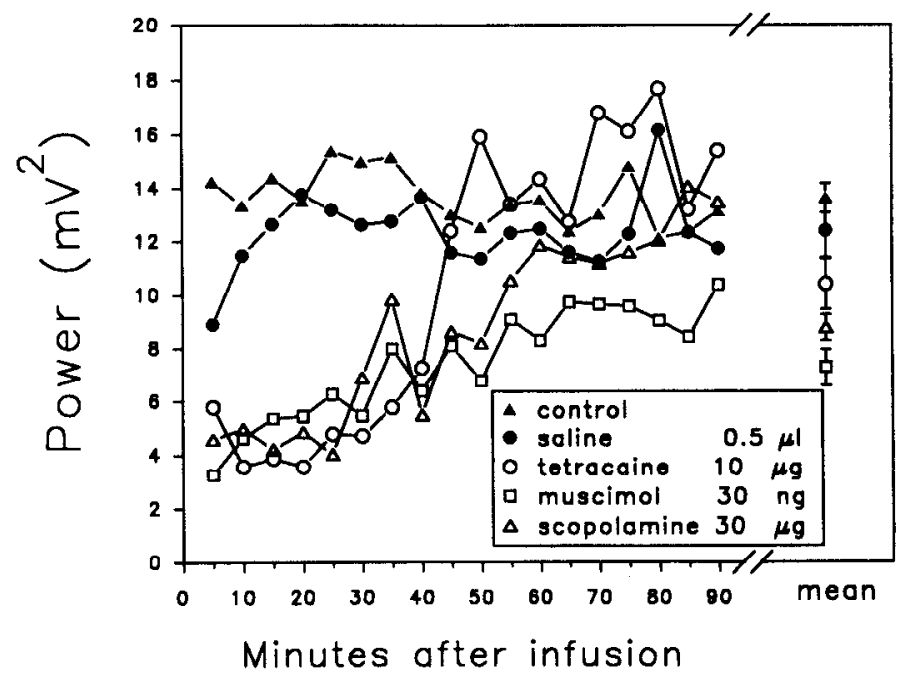

Figure 4. Hippocampal theta rhythm following MSA infusions during pertormance of the CCD. The sessions are the same as those in Figure 3 . The mean level of theta for the entire session is given on the right. Both drug and time after infusion had significant effects on theta.

significantly correlated following each drug for each of the three delays. The correlation was greatest at the $20 \mathrm{sec}$ ISI $(r=0.61$, $p<0.01$; Fig. 5), but was also significant at the $10 \mathrm{sec}$ and 2.5 sec ISIs $(r=0.53, p<0.05$ and $r=0.41, p<0.05$, respectively). A third-order (cubic) function provided the best fit to the data for the change over time in both physiology and behavior.

Hemicholinium binding. MSA infusions of muscimol (15 ng) selectively reduced the density $\left(B_{\max }\right)$ of ${ }^{3} \mathrm{H}$-hemicholinium-3 binding in the hippocampus $(t=-2.61, p<0.05)$, but not in the striatum $(t=-0.16, p>0.05)$ or frontal cortex $(t=1.05$, $p>0.05)$. The decrease in the entorhinal cortex was substantial, but did not reach significance $(t=-1.69, p=0.06$; Table 1$)$.

\section{NBM infusions}

In three rats, the lowest dose of muscimol ( $5 \mathrm{ng}$ ) or scopolamine $(5 \mu \mathrm{g})$ was infused into the NBM prior to testing. In all cases, the rats stopped responding entirely, and thus no further NBM infusions were given to any rats trained in the CCD.

\section{Sensory discrimination (SD)}

Acquisition was rapid, and the mean number of sessions to reach criterion performance was $12 \pm 2.2$. Within a daily session, choice accuracy was stable during the entire $90 \mathrm{~min}$ session. The ISI did not affect choice accuracy $\left(F_{2.60}=0.67, p>0.05\right)$, which was, respectively, $99.3( \pm 0.2), 99.3( \pm 0.2)$, and 99.0 $( \pm 0.2)$ at $2.5,10$, and $20 \mathrm{sec}$ ISIs.

Measures of general task performance were stable within subjects across daily sessions. Responding during the ISI was low with a mean of $0.6( \pm 0.1)$ per trial, and response omissions were infrequent, occurring on only $4.3 \%( \pm 1.2)$ of the trials.

\section{$M S A$ infusions}

Behavior. A total of 89 infusions was given to 14 rats $(6.4 \pm$ 0.8 infusions/rat). Choice accuracy was unaffected (no main effect of drug, $F_{2.87}=0.64, p>0.05$; Table 2).

Theta. Drug infusion had a significant effect on hippocampal theta $\left(F_{2.72}=7.3, p<0.01\right.$; Fig. 6 , right). Muscimol $(30 \mathrm{ng})$ and scopolamine $(30 \mu \mathrm{g})$ reduced the power of theta over the entire 90 min session, and lower doses of muscimol (10 ng) and sco-

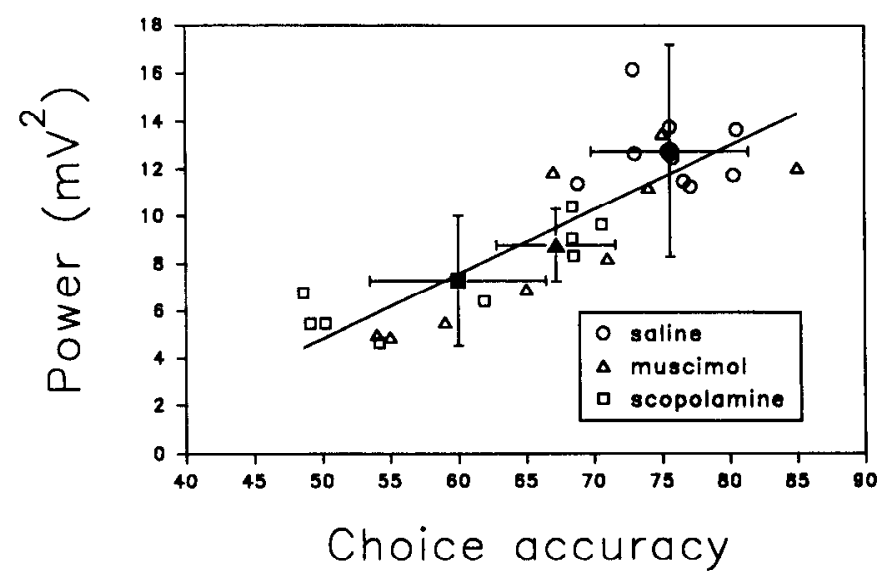

Figure 5. Correlation of choice accuracy at the longest ( $20 \mathrm{sec}$ ) ISI in the CCD and spectral power in the theta frequency range. The positive correlation between these measures was significant $(r=0.61, p<0.01)$. The data in this figure are taken from Figure 3 (20 sec ISI) and Figure 4. Each symbol represents the group mean for a 10 min interval of testing, producing nine data points for each of the substances that were infused: saline, muscimol (30 ng), and scopolamine (30 $\mu \mathrm{g})$.

polamine $(15 \mu \mathrm{g})$ also reduced theta, but only during the first $30 \mathrm{~min}$.

\section{NBM infusions}

Behavior. Twelve rats that received MSA infusions also received NBM infusions. Choice accuracy was significantly reduced by drug infusion $\left(F_{2,60}=3.26, p<0.05\right)$. Saline had no effect on performance as compared to baseline. Scopolamine and muscimol caused dose-dependent decreases in choice accuracy ( $p$ $<0.05$; Table 2, Fig. 6, left).

Different drugs had different effects on response omissions or ISI responses as indicated by an interaction of drug infusions and these behavioral measures. Post hoc analysis revealed that muscimol (30 ng) increased response omissions, and scopolamine decreased $(5 \mu \mathrm{g})$ or increased $(30 \mu \mathrm{g})$ response omissions and increased ISI responses $(5,15$, and $30 \mu \mathrm{g})$. However, changes in choice accuracy occurred during the first half of the test session, whereas changes in response omissions occurred during the last half of each session (Table 2).

Theta. Infusions did not change theta activity (Fig. 6, right), and the correlation between theta and choice accuracy was not significant $(r=0.18, p>0.05)$.

\section{Discussion}

Septohippocampal system

Working and reference memory

Three dissociations in the present cxperiment suggest that the septohippocampal system is important for working memory but

Table 1. ${ }^{3}$ H-hemicholinium-3 binding ( $B_{\max }$ values) $10 \mathrm{~min}$ after intraseptal microinfusion of saline $(0.5 \mu \mathrm{l})$ or muscimol (15 $\mathrm{ng})$, expressed as mean $\pm \mathrm{SEM} \mathbf{f m o l} / \mathrm{mg}$ protein

\begin{tabular}{lll} 
Brain region & Saline $(0.5 \mu \mathrm{l})$ & Muscimol $(15 \mathrm{ng})$ \\
\hline Dorsal hippocampus & $21.1 \pm 4.3$ & $11.4^{*} \pm 0.7$ \\
Entorhinal cortex & $26.3 \pm 2.5$ & $20.5 \pm 1.3$ \\
Frontal cortex & $35.0 \pm 2.3$ & $44.2 \pm 6.9$ \\
Striatum & $89.0 \pm 12.2$ & $85.4 \pm 13.3$
\end{tabular}

*p<0.05. 


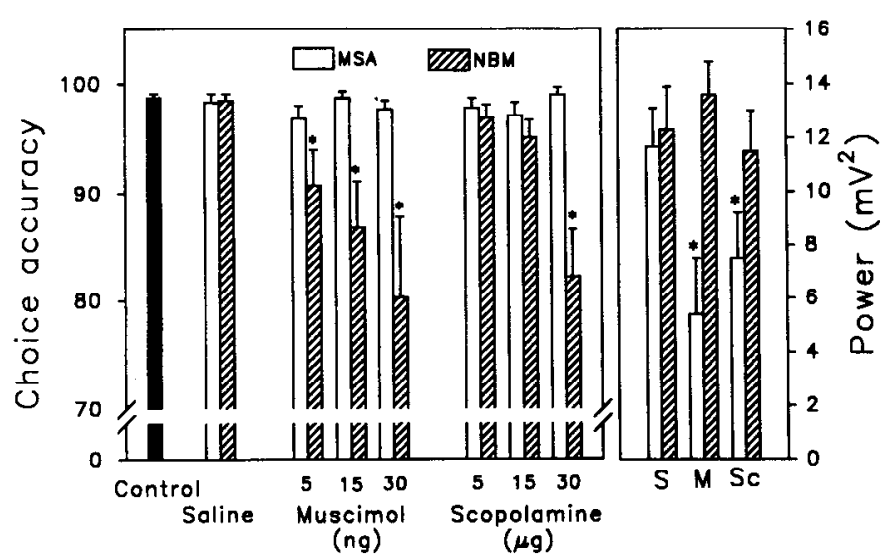

Figure 6. Choice accuracy and theta rhythm in the sensory discrimination (SD) as a function of drug and site of infusion. Infusions into the MSA had no effect on choice accuracy, but suppressed the theta rhythm. Microinfusions into the NBM dose-dependently reduced choice accuracy, but had no effect on theta rhythm. ${ }^{*}, p<0.05$.

not for reference memory: (1) the impairment of choice accuracy in the CCD, but not the SD; (2) in the CCD, the impairment in choice accuracy, but not in response omissions or ISI responses; and (3) the interaction in the magnitude of the impairment in the CCD with the length of the ISI.

Microinfusion of muscimol or scopolaminc into the MSA severely impaired choice accuracy in the CCD, but not in the SD. All elements of the behavioral tasks were the same except the mnemonic demands. Both tasks used identical stimuli, responses, motivation, reinforcement, order of the stimuli, and ISIs; the only difference between the CCD and the SD was the requirement for working memory in the former but not the latter. Therefore, the common elements between CCD and SD could not have been responsible for the selective impairment in the $\mathrm{CCD}$ as compared to the SD. Rather, the unique component of the $\mathrm{CCD}$, the requirement for trial-dependent working memory, must have been the variable that required the septohippocampal system. Similar results in a variety of other tasks, in which the septohippocampal system was critical for working, but not reference memory, have followed septal lesions (Gray and McNaughton, 1983; Kesner et al., 1986; Miyamoto et al., 1987; Hagan et al., 1988).

A second dissociation is the differential effects of intraseptal infusions on performance within the CCD. The behavioral measures included one that assessed working memory (choice accuracy) and two others that assessed reference memory (response omissions, ISI responses). Except for the first few blocks of trials during a test session, the infusions that severely impaircd working memory, as reflected by significant decreases in choice accuracy, had no effect on reference memory, as indicated by normal scores for response omissions and ISI responses. The most parsimonious explanation of this dissociation is that the septohippocampal system is critical for working memory but not reference memory.

A third dissociation supports the same conclusion. The significant interaction between the ISI and drug was due to a greater impairment of choice accuracy as the ISI increased. For example, following intraseptal tetracaine, choice accuracy for the shortest ISI was normal, and choice accuracy at the longer ISIs was impaired. Except for the demand on working memory, the components of the task at the short ISI were the same as those at the longer ISIs. Consequently, the variation in the impairment of choice accuracy across ISIs must be due to the task component that varied as a function of the ISI, which was the demand for working memory.

The level of interference and the conditional rules governing the CCD task may have contributed to the working memory impairment following intraseptal infusion. The amount of interference the rat encountered from previous stimuli when evaluating current stimuli was substantial because there were only two stimuli. When the data were analyzed for trials in which the previous trial contained the same stimulus but opposite response requirements, proactive interference was present. Choice accuracy on match trials preceded by a nonmatch trial was lower than on match trials preceded by a match trial (data not shown). The high level of interference that is inherent in working memory tasks may be the element that requires normal functioning of the septohippocampal system for accurate per-

Table 2. Behavioral performance in the sensory discrimination (SD) task following microinfusions into the NBM or MSA

\begin{tabular}{|c|c|c|c|c|c|c|}
\hline \multirow[b]{2}{*}{ Drug } & \multicolumn{2}{|c|}{ Choice accuracy } & \multicolumn{2}{|c|}{ Response omissions } & \multicolumn{2}{|l|}{ ISI responses } \\
\hline & MSA & NBM & MSA & NBM & MSA & NBM \\
\hline Control & $98.8 \pm 0.3$ & & $4.3 \pm 0.3$ & & $0.6 \pm 0.1$ & \\
\hline Saline & $98.2 \pm 0.8$ & $98.2 \pm .7$ & $4.0 \pm 0.3$ & $4.3 \pm 0.4$ & $0.61 \pm 0.13$ & $0.76 \pm 0.21$ \\
\hline \multicolumn{7}{|c|}{ Muscimol } \\
\hline $5 \mathrm{ng}$ & $96.6 \perp 1.2$ & $91.6 \perp 3.2$ & $3.5 \perp 0.4$ & $3.5 \pm 0.7$ & $0.78 \pm 0.24$ & $0.79 \pm 0.24$ \\
\hline $15 \mathrm{ng}$ & $98.5 \pm 0.7$ & $82.1 \pm 5.3^{*}$ & $3.2 \pm 0.6$ & $6.4 \pm 1.2$ & $0.39 \pm 0.08$ & $0.56 \pm 0.23$ \\
\hline $30 \mathrm{ng}$ & $98.1 \pm 0.7$ & $78.6 \pm 7.8^{*}$ & $3.9 \pm 0.3$ & $8.9 \pm 1.9^{*}$ & $0.89 \pm 0.30$ & $0.47 \pm 0.36$ \\
\hline \multicolumn{7}{|c|}{ Scopolamine } \\
\hline $5 \mu \mathrm{g}$ & $98.0 \pm 0.8$ & $97.3 \pm 1.1$ & $2.8 \pm 0.8$ & $1.1 \pm 2.0^{*}$ & $0.78 \pm 0.37$ & $1.50 \pm 1.26^{*}$ \\
\hline $15 \mu \mathrm{g}$ & $97.8 \pm 1.0$ & $95.1 \pm 1.8$ & $3.9 \pm 0.4$ & $2.7 \pm 1.1$ & $0.90 \pm 0.37$ & $1.26 \pm 0.32 *$ \\
\hline $30 \mu \mathrm{g}$ & $98.8 \pm 0.8$ & $80.5 \pm 4.1^{*}$ & $6.8 \pm 2.4$ & $8.3 \pm 1.4^{*}$ & $0.30 \pm 0.11^{*}$ & $1.09 \pm 0.42^{*}$ \\
\hline
\end{tabular}

Data are given as the mean \pm SEM, with choice accuracy and response omissions as percentage of total trials, and interstimulus interval (ISI) responses as the number of responses per trial. Analysis of each 10 min time interval revealed that changes in choice accuracy occurred during the first $40 \mathrm{~min}$ of the session, whereas changes in response omissions occurred during the last $\mathbf{4 0} \mathrm{min}$, and changes in ISI responses occurred throughout the session.

${ }^{*} p<0.05$. 
formance (Olton and Shapiro, 1993; Shapiro and Olton, 1994). Delay tasks with larger numbers of discriminative stimuli, and thus less interference, are less sensitive to septohippocampal disruption (Otto and Eichenbaum, 1992; Rawlins et al., 1993). Moreover, the CCD is different from most delay nonmatch-tosample tasks in that it requires the retrieval of different response rules for match and nonmatch conditions. Tasks that require conditional discriminations may be more sensitive to septohippocampal disruption (see Shaw and Aggleton, 1993).

\section{Spatial and nonspatial memory}

The decrease in choice accuracy following intraseptal infusions indicates an impairment in nonspatial working memory because the relevant stimulus dimensions in the CCD task were nonspatial. Previous work from this laboratory, in which identical microinfusion procedures were used, demonstrated that the septohippocampal system is also important for spatial working memory (Givens and Olton, 1990; Givens et al., 1992). Numerous studies with septal lesions have shown the importance of the septohippocampal system for spatial working memory (Harrell et al., 1987; Kelsey and Landry, 1988; Mizumori et al., 1990; Numan and Quaranta, 1990; Decker et al., 1992), and some have demonstrated its involvement in nonspatial working memory (Olton and Feustle, 1981; Dunnett, 1985; Meck ct al., 1987; Kesner et al., 1988). Taken together with the present data, these studies indicate that irrespective of stimulus type, testing apparatus, or experimental manipulation, working memory is impaired when the septohippocampal system is compromised.

The only relevant spatial aspect of the CCD and SD tasks was the location of the response levers. If intraseptal infusions selectively disrupt spatial processing, and not working memory, intraseptal infusions should have affected spatial response selection equally in both tasks. They did not; only the CCD task was affected. Thus, the septohippocampal system is not required for simple selection of the spatial response. However, disruption of spatial processing by intraseptal infusions may become manifest under the more complex discrimination conditions of the CCD. The hypothesis that with conditional discriminations, septohippocampal disruption selectively affects responses requiring a significant spatial component could be tested with the use of "nonspatial" (go/no-go) response rules.

\section{Hippocampal theta}

The time-dependent changes in theta rhythm were correlated with changes in working memory. Hippocampal theta has long been associated with mnemonic processes (Landfield et al., 1971; Berry and Thompson, 1978; Winson, 1978; Rawlins, 1985), and was highly correlated with accuracy on both classical and instrumental conditioning tasks. The present data confirm the importance of theta activity to accurate performance, and indicate that when theta activity was suppressed by intraseptal infusion, choice accuracy was also impaired. The two measures were temporally linked. As theta recovered, performance at the shortest delay recovered first, and choice accuracy at the longest delay did not return to baseline levels until theta was normal. Regression analysis revealed that a cubic function best describes both the recovery of theta and the return of accuracy at all three delay intervals, indicating that a common mechanism may underlie these processes, and that working memory may be dependent on theta activity, or neural mechanisms associated with it.

The septohippocampal pathway may entrain the hippocam- pus to retain trial-dependent information by enhancing or potentiating circuits carrying trial-specific sensory information. Trial-independent performance that is overtrained may not involve such a mechanism. Theta may be involved in the synaptic strengthening of connections in the hippocampus through the processes of long-term potentiation (Larson and Lynch, 1986; see also Eichenbaum et al., 1992). The present work, demonstrating that intrascptal infusions disrupt choice accuracy in a working but not reference memory task, even though theta activity is affected equally in the two tasks, suggests that hippocampal theta is critical for working memory, perhaps via a mechanism that involves LTP.

\section{Acetylcholine}

Hippocampal cholinergic activity, as indicated by the binding of ${ }^{3} \mathrm{H}$-hemicholinium-3, was decreased following intraseptal infusion of muscimol. Unlike the hippocampus, which receives cholinergic innervation from the MSA, brain regions not innervated by MSA (frontal cortex, striatum) did not show changes in cholinergic activity. Consistent with this interpretation, the entorhinal cortex, which had a marginal decrease, receives cholinergic projections from the MSA (Mitchell et al., 1982). Although a low dose of muscimol ( $15 \mathrm{ng})$ is sufficient to disrupt working memory, previous studics have not demonstrated that these doses reduce cholinergic activity in the hippocampus (Blaker et al., 1984; Durkin, 1992). In mice, the use of spatial memory increased hippocampal cholinergic activity, and intraseptal muscimol $(20 \mathrm{ng})$ prevented this increase. Intraseptal infusions of muscimol produced reductions in hippocampal ACh release as measured by in vivo microdialysis (L. K. Gorman, unpublished observations). The extent to which MSA infusions alter the activity of other neurotransmitter systems remains to be determined.

Loss of hippocampal cholinergic function may be the basis for the working memory impairments following peripheral administration of cholinergic drugs. Scopolamine impaired choice accuracy in the CCD (Spencer et al., 1985; Sakurai and Wenk, 1990), as well as in an operant task of working memory that uses a discrete trial procedure (Dunnett et al., 1991). The present data suggest that the amnestic effects of anticholinergic drugs may be due to their action on cholinergic receptors in the MSA (Bialowas and Frotscher, 1987; see Givens and Olton, 1992). Further intracranial infusion studies are needed to demonstrate the relative contribution of multiple sites within the CNS to changes in mnemonic processes following systemic cholinergic drugs.

\section{Nucleus basalis magnocellularis}

The decrease in choice accuracy in the SD following microinfusion of muscimol into the NBM suggests that the NBM has a role in reference memory. Mnemonic analysis of the functions of the NBM are relatively recent and largely based on neurotoxic lesions (see Olton and Wenk, 1987, for a review). The functional role of the NBM may become increasingly important as the cognitive demands increase in the task. The lowest dose of muscimol $(5 \mathrm{ng}$ ) into the NBM completely disrupted CCD choice accuracy but had no substantial effect on any measures of SD performance. The SD had a higher level of choice accuracy than CCD (Fig. 3), and was perhaps less sensitive to chemical disruption. In the NBM, the highest dose of muscimol $(30 \mathrm{ng})$, and all doses of scopolamine, affected response omissions and ISI responses. Response time decreased following muscimol infu- 
sions into the NBM in a similar discrimination task (Dudchenko and Sarter, 1991). Response time was not recorded in the present experiment, but if decreased, would impact working memory processes more significantly than reference memory processes. Changes in non-mnemonic behavioral measures indicate that attentional/motivational aspects of task performance may also be affected by the NBM.

\section{The basal forebrain and memory}

Neural function in the MSA and NBM may be differentially regulated by $\mathrm{GABA}$ and $\mathrm{ACh}$ as a function of their anatomical distribution with the basal forebrain. The density of GABA innervation is greater than that of cholinergic innervation in both the MSA and NBM. The results indicate that GABAergic innervation of the MSA may have a greater influence on memory than cholinergic innervation. Intraseptal muscimol had a longer-lasting effect than scopolamine on both accuracy and theta. Likewise, mnemonic performance in the SD was more affected by muscimol than by scopolamine. Conversely, nonmnemonic task parameters (response omissions and interstimulus interval responses) were more affected by scopolamine, suggesting a nonspecific or nonselective effect of scopolamine in the NBM (e.g., on cerebral blood flow; see Armstrong, 1986). Muscimol had a selective effect on choice accuracy when infused into either the MSA or NBM. The data fit with anatomical evidence that indicates GABA innervation is well established in both the MSA and NBM, but that cholinergic innervation is less cxtensive (Ingham et al., 1988; Záborszky et al., 1991).

The anatomy of the basal forebrain is highly complex and contains a multitude of pathways potentially subserving memory. The cholinergic projection to the hippocampus is not the only pathway originating in MSA that may mediate the effects of intraseptal infusions. For example, a sizeable proportion of the septohippocampal pathway consists of GABAergic neurons (Kohler et al., 1984; Wainer et al., 1985) that terminate on interneurons in the hippocampus (Freund and Antal, 1988). Second, a large number of descending projections from the MSA terminate in the hypothalamus and brainstem, but their function is poorly understood. Third, ascending projections from the MSA terminate not only in hippocampus, but in cingulate, entorhinal, and subicular cortices where they also produce rhythmic oscillations that have been linked to mnemonic processes (Mitchell et al., 1982; Sutherland et al., 1988). The trend toward a decrease in ${ }^{3} \mathrm{H}$-hemicholinium-3 binding in the entorhinal cortex indicates that intraseptal infusions may affect the septoentorhinal projection, and, as a consequence, influence spatial memory (Mizumori et al., 1992). An important goal of future research is the identification of the role of these and other basal forebrain circuits during acquisition, retention, and recall. Ultimately, an understanding of these pathways and processes may provide new insights into human memory and into amnesia that is due to pathology in these systems (Baddeley et al., 1991; Gaffan and Gaffan, 1991).

\section{References}

Armstrong DM (1986) Ultrastructural characterization of choline acetyltransferase-containing neurons in the basal forebrain of rat: evidence for a cholinergic innervation of intracerebral blood vessels. $J$ Comp Neurol 250:81-92.

Baddeley AD, Bressi S, Della Sala S, Logie R, Spinnler H (1991) The decline of working memory in $\Lambda$ lzheimer's disease. $\Lambda$ longitudinal study. Brain 114:2521-2542.

Barnes CA (1988) Spatial learning and memory processes: the search for their neurobiological mechanisms in the rat. Trends Neurosci 11: 163-169.

Rerry SD, Thompson RF (1978) Prediction of learning rate from the hippocampal electroencephalogram. Science 200:1298-1300.

Bialowas J, Frotscher M (1987) Choline acetyltransferase-immunoreactivity neurons and terminals in the rat septal complex: a combincd light and electron microscope study. J Comp Neurol 259:298-307.

Blaker WD, Peruzzi G, Costa E (1984) Behavioral and neurochemical differentiation of specific projections in the septal-hippocampal cholinergic pathway in the rat. Proc Natl Acad Sci USA 81:1880-1882.

Brioni JD, Decker MW, Gamboa LP, Izquierdo I, McGaugh JL (1990) Muscimol injections in the medial septum impair spatial learning. Brain Res 522:227-2.34.

Buzsaki G, Bickford RG, Ponomereff G, Thal LJ, Mandel R, Gage FH (1988) Nucleus basalis and thalamic control of neocortical activity in the freely moving rat. J Neurosci 8:4007-4026.

Chrobak JJ, Napier TC (1992) Antagonism of GABAergic transmission within the septum disrupts working/episodic memory in the rat. Neuroscience 47:833-841.

Chrobak JJ, Stackman RW, Walsh TJ (1989) Intraseptal administration of muscimol produces dose-dependent memory impairments in the rat. Behav Neural Biol 52:357-369.

Decker MW, Radek RJ, Majchrzak MJ, Anderson DJ (1992) Differential effects of medial septal lesions on spatial-memory tasks. Psychobiology 20:9-17.

Dudchenko P, Sarter M (1991) GABAergic control of basal forebrain cholinergic neurons and memory. Behav Brain Res 42:33-41.

Dunnett SB (1985) Comparative effects of cholinergic drugs and nucleus basalis or fimbria-fornix lesions on delayed matching to sample in rats. Psychopharmacology 87:357-363.

Dunnett SB, Fveritt BJ, Robbins TW (1991) The basal forebraincortical cholinergic system: interpreting the functional consequences of excitotoxic lesions. Trends Neurosci 14:494-501.

Durkin TP (1992) G $\Lambda \mathrm{B} \Lambda$ ergic mediation of indirect transsynaptic control over basal and spatial memory testing-induced activation of septo-hippocampal cholinergic activity in mice. Behav Brain Res 50: 155-165.

Eichenbaum H, Otto T, Cohen NJ (1992) The hippocampus-what does it do. Behav Neural Biol 57:2-36.

Freund TF, Antal M (1988) GABA-containing neurons in the septum control inhibitory interneurons in the hippocampus. Nature 336:170173.

Gaffan D, Gaffan EA (1991) Amnesia in man following transection of the fornix. A review. Brain 114:2611-2618.

Givens B, Olton DS (1990) Cholinergic and GABAergic modulation of medial septal area: effect on working memory. Behav Neurosci 104:849-855.

Givens B, Olton D (1992) Working memory and cholinergic autoregulation in the medial septal area. In: Neurotransmitter interactions and cognitive function (Levin ED, Decker MW, Butcher LL, eds), pp 301311. Boston: Birkhauser.

Givens B, Olton DS, Crawley J (1992) Galanin in the medial septal area impairs working memory. Brain Res 582:71-77.

Gray JA, McNaughton N (1983) Comparison of behavioral effects of septal and hippocampal lesions: a review. Neurosci Biobehav Rev 7:119-188.

Hagan JJ, Salamone JD, Simpson J, Ivesen SD, Morris RGM (1988) Place navigation in rats is impaired by lesions of medial septum and diagonal hand but not nucleus hasalis magnocellularis. Behav Brain Res 27:9-20.

Harrell LE, Barlow TS, Parsons D (1987) Cholinergic neurons, learning, and recovery of function. Behav Neurosci 101:644 652 .

Ingham CA, Bolam JP, Smith AD (1988) GABA-immunoreactive synaptic boutons in the rat basal forebrain: comparison of neurons that project to the neocortex with pallidosubthalamic neurons. J Comp Neurol 273:263-282.

Kelsey JE, Landry BA (1988) Medial septal lesions disrupt spatial mapping ability in rats. Behav Neurosci 102:289-293.

Kesner RP, Crutcher KA, Measom MO (1986) Medial septal and nucleus basalis magnocellularis lesions produce order memory deficits in rats which mimic symptomatology of Alzheimer's disease. Neurobiol Aging 7:287-295.

Kesner RP, Crutcher K, Beers DR (1988) Serial position curves for item information: role of the dorsal hippocampal formation and medial septum. Brain Res 454:219-226. 
Kohler C, Chan-Palay V, Wu J-Y (1984) Septal neurons containing glutamic acid decarboxylase immunoreactivity project to the hippocampal region in the rat brain. Anat Embryol (Berl) 169:41-44.

Landfield PW, McGaugh JL, Tusa RJ (1971) Theta rhythm: a tcmporal correlate of memory storage processes in the rat. Science 175: 87-89.

Larson J, Lynch G (1986) Induction of synaptic potentiation in hippocampus by patterned stimulation involves two events. Science 232: 985-988.

Lewis PR, Shute CCD (1967) The cholinergic limbic system: projections to hippocampal formation, medial cortex, nuclei of the ascending cholinergic reticular formation, and the subfornical organ and supraoptic crest. Brain 90:521-542.

Markram H, Segal M (1990) Long-lasting facilitation of excitatory postsynaptic potentials in the rat hippocampus by acetylcholine. $J$ Physiol (Lond) 427:381-393.

Meck WH, Church RM, Wenk GL, Olton DS (1987) Nucleus basalis magnocellularis and medial septal area lesions differentially impair temporal memory. J Neurosci 7:3505-3511.

Mesulum MM, Mufson EJ, Wainer BH, Levey AI (1983) Central cholinergic pathways in the rat: an overview based on an alternative nomenclature. Neuroscience 10:1185-1201.

Milner TA (1991) Cholinergic neurons in the rat septal complex: ultrastructural characterization and synaptic relations with catecholaminergic terminals. J Comp Neurol 314:37-54.

Mitchell S.I, Rawlins JNP, Steward O, Olton DS (1982) Medial septal area lesions disrupt theta rhythm and cholinergic staining in medial entorhinal cortex and produce impaired radial arm maze behavior in rats. J Neurosci 2:292-302.

Miyamoto M, Kata J, Narumi S, Nagaoka A (1987) Characteristics of memory impairments following lesioning of the basal forebrain and the medial septal nucleus in rats. Brain Res 419:19-31.

Mizumori SJY, Perez GM, Alvarado MC, Barnes CA, McNaughton BL (1990) Reversible inactivation of the medial septum differentially affects two forms of learning in rats. Brain Res 528:12-20.

Mizumori SJY, Ward KE, Lavoie AM (1992) Medial septal modulation of entorhinal single unit activity in anesthetized and freely moving rats. Brain Res 570:188-197.

Muir JL, Robbins TW, Everitt BJ (1992) Disruptive effects of muscimol infused into the basal forebrain on conditional discrimination and visual attention: differential interactions with cholinergic mechanisms. Psychopharmacology 107:541-550.

Nagahara AH, McGaugh JL (1992) Muscimol infused into the medial septal area impairs long-term memory but not short-term memory in inhibitory avoidance, water maze place learning and rewarded alternation tasks. Brain Res 591:54-61.

Numan R, Quaranta JR Jr (1990) Effects of medial septal lesions on operant delayed alternation in rats. Brain Res 531:232-241.

Olton DS, Feustle WA (1981) Hippocampal function required for nonspatial working memory. Exp Brain Res 41:380-389.

Olton DS, Shapiro ML (1993) Mnemonic dissociations: the power of parameters. J Cognit Neurosci 4:200-207.

Olton DS, Wenk GL (1987) Dementia: Animal models of the cognitive impairments produced by degeneration of the basal forebrain cholinergic system. In: Psychopharmacology: the third generation of progress (Meltzer HY, ed), pp 941-953. New York: Raven.
Olton DS, Givens BS, Markowska AM, Shapiro M, Golski S (1992) Mnemonic functions of the cholinergic septohippocampal system. In: Memory: organization and locus of change (Squire LR, Weinberger NM, Lynch G, McGaugh JL, eds), pp 250-269. New York: Oxford.

Otto T, Eichenbaum H (1992) Complementary roles of the orbital prefrontal cortex and the perirhinal-entorhinal cortices in an odorguided delayed-nonmatching-to-sample task. Behav Neurosci 106: $762-775$.

Paxinos G, Watson C (1986) The rat brain in stereotaxic coordinates, 2d ed. New York: Academic.

Pontecorvo MJ (1983) Effects of proactive interference on rats' continuous nonmatching-to-sample performance. Anim Learn Behav 11: 356-366.

Rawlins JNP (1985) Some neurophysiological properties of the septohippocampal system. In: Psychopharmacology of the limbic system (Trimble MR, Zarifian E, eds), pp 17-50. New York: Oxford.

Rawlins JNP, Lyford GL, Seferiades A, Deacon RMJ, Cassiday HJ (1993) Critical determinants of nonspatial working memory deficits in rats with conventional lesions of the hippocampus or fornix. Behav Neurosci 107:420-433.

Richardson RT, DeLong MR (1990) Context-dependent responses of primate nucleus basalis neurons in a go/no-go task. J Neurosci 10: 2528-2540.

Robbins TW, Everitt BJ, Marston HM, Wilkinson J, Jones GH, Page KJ (1989) Comparative effects of ibotenic acid and quisqualic acidinduced lesions of the substantia innominata on attentional function in the rat: further implications for the role of the cholinergic neurons of the nucleus basalis in cognitive processes. Behav Brain Res 35: $221-240$

Sakurai Y, Wenk GL (1990) The interaction of acetylcholinergic and serotonergic systems on performance in a continuous nonmatching to sample task. Brain Res 519:118-121.

Shapiro ML, Olton DS (1994) Hippocampal function and interference. In: Memory systems, 1994, (Schacter DL, Tulving E, eds), in press. Cambridge, MA: MIT Press.

Shaw C, Aggleton JP (1993) The effects of fornix and medial prefrontal lesions on delayed non-matching-to-sample by rats. Behav Brain Res 54:91-102.

Spencer DG, Pontecorvo MJ, Heise GA (1985) Central cholinergic involvement in working memory: effects of scopolamine on continuous nonmatching and discrimination performance in the rat. Behav Neurosci 99:1049-1065.

Sutherland R J, Whishaw IQ, Kolb B (1988) Contributions of cingulate cortex to two forms of spatial learning and memory. J Neurosci 8:18631872.

Wainer J $\Lambda$, Levey $\Lambda \mathrm{I}$, Rye DB, Mesulam M-M, Mufson EJ (1985) Cholinergic and non-cholinergic septohippocampal pathways. Neurosci Lett 54:45-52.

Wan RQ, Pang K, Olton D (1990) Hippocampus and working memory: continuous conditional discrimination and T-maze alternation. Soc Neurosci Abstr 16:113.4.

Winson J (1978) Loss of hippocampal theta rhythm results in spatial memory deficit in the rat. Science 210:160-163.

Záborszky L, Cullinan WE, Braun A (1991) Afferents to basal forebrain cholinergic projection neurons: an update. Adv Exp Med Biol 295: 43-100. 